\title{
COVID-19 - Protocol for Observational Studies Utilising Near Real-Time Electronic Australian General Practice Data to Promote Effective Care and Best-Practice Policy - A Design Thinking Approach
}

\author{
Andrew Georgiou ( $\square$ andrew.georgiou@mq.edu.au ) \\ Macquarie University https://orcid.org/0000-0002-7619-3668 \\ Julie Li \\ Macquarie University \\ Christopher Pearce \\ Outcome Health \\ Adam McLeod \\ Outcome Health \\ Nasir Wabe \\ Macquarie University \\ Rae-Anne Hardie \\ Macquarie University \\ Guilherme Saffi Franco \\ Macquarie University \\ Chisato Imai \\ Macquarie University \\ Gorkem Sezgin \\ Macquarie University \\ Judith Thomas \\ Macquarie University \\ Zhaoli Dai \\ Macquarie University \\ Muhammad Kashif Sheikh \\ Gippsland Primary Health Network \\ Amanda Proposch \\ Gippsland Primary Health Network
}

\section{Stephen Weeding}

South Eastern Melbourne Primary Health Network

\section{Brendon Wickham}

South Eastern Melbourne Primary Health Network

\section{Tony Badrick}

Royal College of Pathologists of Australasia, Quality Assurance Programs

Darnel Murgatroyd

Digital Health Cooperative Research Centre 


\section{Study protocol}

Keywords: COVID-19, general practice, design thinking, quality of care, observational studies, pandemics

Posted Date: December 31st, 2020

DOI: https://doi.org/10.21203/rs.3.rs-136810/v1

License: (c) (i) This work is licensed under a Creative Commons Attribution 4.0 International License. Read Full License 


\section{Abstract}

Background: Health systems around the world have been forced to make choices about how to prioritise care, manage infection control and maintain reserve capacity for future disease outbreaks. Primary health care has moved into the frontline as COVID-19 testing transitions from hospitals to multiple providers, where tracking testing behaviours can be fragmented and delayed. Pooled general practice data are a valuable resource which can be used to inform population and individual care decision-making. This project aims to utilise near real-time electronic general practice data to promote effective care and best-practice policy.

Methods: The project will utilise a design thinking approach involving all collaborators (Primary Health Networks [PHNs], general practices, consumer groups, researchers, and digital health developers, pathology professionals) to enhance the development of meaningful and translational project outcomes. The project will be based on a series of observational studies utilising near real-time electronic general practice data from a secure and comprehensive digital health platform [POpulation Level Analysis and Reporting (POLAR) general practice data warehouse]. The study will be carried out over 1.5 years (July 2020 - December 2021) using data from over 350 general practices within three Victorian Primary Health Networks (PHNs) and Gippsland PHN, Eastern Melbourne PHN and South Eastern Melbourne PHN, supplemented by data from consenting general practices from two PHNs in New South Wales, Central and Eastern Sydney PHN and South Western Sydney PHN.

Discussion: Developed using a design thinking approach, this project will deliver: 1) A near real-time geo-spatial reporting framework at community, state and nation-wide levels to identify emerging trends and monitor the impact of interventions/policy decisions. 2) Timely evidence about the impact of the COVID-19 pandemic related to its diagnosis, treatment and medications prescribed and its impact on patients. 3) A predictive geo-spatial analytics dashboard for timely, evidence-based decision-making at community, state and nation-wide levels. 4) An evidence-based suite of general practice outcome measures to monitor incidence, prevalence, recovery and mortality in response to the COVID19 pandemic.

\section{Contributions To The Literature}

- Pooled near real-time general practice data are a valuable resource which can be used to inform care decisionmaking at the individual and population level.

- A design thinking approach in the development of the research process seeks to incorporate user needs and feedback throughout to enhance the development of meaningful and translational project outcomes.

- This project will demonstrate the use of general practice data in monitoring the quality and effectiveness of care to inform and support health planning, including pandemic responses.

\section{Background}

Since its identification in December 2019, SARS-CoV-2 and its associated coronavirus disease (COVID-19) has had a devastating effect on communities around the world, leading to a rapidly escalating mortality toll of over a million people[2]. Health systems around the world have been forced to make rapid choices about how to prioritise care, manage infection control and maintain reserve capacity for future disease outbreaks[3]. The interruption of normal patterns of health care and the suspension of services has meant that the pandemic has also had a major impact on the detection and treatment of many non-COVID-19 conditions[4].

The health care consequences associated with the COVID-19 pandemic range from people's avoidance of contact with health care settings either for fear of contracting COVID-19, or as a means of reducing pressure on the health system[5], 
or because of the increased financial stress caused by the pandemic[6]. Early evidence from an Australian Bureau of Statistics Household Impacts of COVID-19 Survey showed that one in 14 Australians (1.4 million adults) were unable to see their general practitioner (GP) or other health professional in person during the early April 2020 to early May 2020 period[7]. Australian Medicare figures revealed that pathology testing fell by almost 30 per cent; consultations with specialists also fell by eight per cent; and medical operations by 27 per cent[8]. The pandemic has had a disproportionate impact on disadvantaged communities helping to entrench inequalities, as a consequence of the economic measures used to contain the virus[3]. The slump in general practice visits may have detrimental and longterm effects on patient care and outcomes, especially if it impacts on the diagnosis of new conditions, recommended disease and cancer screening programs, or ongoing monitoring of patients with chronic disease[9, 10].

Whilst the initial focus has been on COVID-19 and its mortality, the effects of SARS-CoV-2 are evolving into (at this stage) a three-pronged disease.

- Acute COVID-19, the disease responsible for most of the initial morbidity and in particular mortality[11]

- Long Covid, the emerging long term effects of SARS-CoV-2 infection, with or without initial hospitalisation[12, 13]

- Paediatric disease, variously called Paediatric Inflammatory Multisystem Syndrome Temporally associated with SARS-COV-2 (PIMS-TS) in Europe and also known as Multisystem Inflammatory Syndrome in Children (MIS-C) in the USA[14].

General practice services have a critical role to play in Australia's response to COVID-19, not least because they are often the place of initial health system contact for most Australians[15]. They are essential as suppliers of information to the community in terms of health care advice to mitigate the spread of disease and protect the health of the community[16]. General practice activity is also key to identifying and monitoring the health of the community, providing an early warning system of the spread of the pandemic and targeting areas where health care may be avoided or delayed, leading to the possibility of missed diagnoses, medications and treatment, which may have serious future consequences for patients and the health care system[8]. While general practices were initially severely affected by a drop in visits post COVID-19 outbreak, the utilisation of Medicare funding for telehealth has helped to reinforce access to general practices and non-GP specialists. Australian Bureau of Statistics figures over a four week period across early April to early May 2020 showed that one in six Australians (17\%) used telehealth services (mostly in the form of telephone calls)[7]. Despite the importance of telehealth initiatives, many patients still require a face-to-face option[17]. In recognition of this, on 30 March 2020 the Commonwealth Government announced temporary increases to incentive payments (Practice Incentives Program Quality Improvement) for general practice to increase support for bulk-billed services with the aim of supporting essential face-to-face care access for patients[18].

Identifying the impact of COVID-19 on the health care system in near real time is an urgent priority for critical decisionmaking about resource allocation, control measures, decision-making, policy development and community communication. Primary health care has moved into the frontline as COVID-19 testing transitions from hospitals to multiple general practice providers where tracking testing behaviours can be fragmented and delayed. Pooled general practice data are a valuable resource which can be used to inform decision-making including individual care, population health and business and government strategy[19].

This paper outlines a project funded by the Digital Health Cooperative Research Centre (DHCRC) which aims to utilise near real-time electronic general practice data to promote effective care and best-practice policy to: a) build a near realtime COVID-19 geo-spatial reporting framework; b) generate timely and critical evidence about the impact of COVID-19; c) build a predictive geo-spatial analytics dashboard for timely, evidence-based decision-making; and d) establish an evidence-based suite of general practice outcome measures required to monitor the quality and effectiveness of care related to incidence and prevalence, recovery and mortality. 
The project was formally identified initially as a Project Concept Proposal to the DHCRC involving a collaboration between Macquarie University, Outcome Health, Gippsland, Eastern Melbourne, South Eastern Melbourne Primary Health Networks and the Royal College of Pathologists of Australasia Quality Assurance Programs. The proposal was assessed by the DHCRC using its Eligibility Threshold Criteria to meet its Research and Investment Framework. Following this we developed a Project Plan which was reviewed by the DHCRC according to its Merit and Value Criteria which led to the establishment of a Project Agreement (17 June 2020).

\section{Methods}

\section{Design Thinking}

The project will utilise a design thinking approach involving all its collaborators (PHNs, general practices, consumer groups, researchers, and digital health developers, pathology professionals) to enhance the development of meaningful and translational project outcomes. Design thinking seeks to incorporate user needs and feedback throughout the development of the research process. The approach involves rounds of ideation, prototyping, and testing[1] to enhance understanding of underlying problems or unpredictable situations[20].

The project builds upon an established collaboration between Macquarie University, the Royal College of Pathologists Quality Assurance Programs, Outcome Health and Gippsland, Eastern and South Eastern PHNs, which evaluated the appropriateness and quality use of pathology in general practice[21] using pooled electronic general practice data[22]. This project provided an overview of pathology testing within Australian general practice, along with reporting on variation across key indicators including vitamin D testing, glycated haemoglobin $(\mathrm{HbA} 1 \mathrm{c})$ and kidney function tests for monitoring type 2 diabetes, ferritin testing for iron deficiency and prostate-specific antigen (PSA) testing[23].

\section{Observational studies}

A series of observational studies will be carried out over 1.5 years (July 2020 - December 2021) utilising near real-time electronic general practice data to promote effective care and best-practice policy. The studies will be centred on 350 general practices within three Victorian PHNs, Gippsland PHN, Eastern Melbourne PHN and South Eastern Melbourne PHN. These PHNs cover metropolitan and rural regions across a combined area totalling 48,903 km, delivering healthcare to 3,132,382 Australians[23]. Data will be supplemented by participation of two PHNs from New South Wales, Central and Eastern Sydney PHN and South Western Sydney PHN. Central and Eastern Sydney PHN encompasses 2,053 general practices and covers 1,637,740 people living in this region[24], while South Western Sydney PHN encompasses 1,045 general practices with 966,450 people living in the region[25].

\section{Data source}

Outcome Health, as a data custodian, uses its Population Level Analysis \& Reporting (POLAR) Data Space to provide a secure and comprehensive digital health platform which collects data from consenting general practices across participating PHNs[19, 21, 22, 26, 27]. Data variables include de-identified demographic information about patients and general practices, as well as visit records (diagnosis, past history, medications, immunisation, radiology) and pathology test records (test name and result).

Until recently, there was little evidence about the landscape of pathology testing and diagnostics in Australian general practice, due to a lack of reliable data and expertise in data management, information systems and quality improvement infrastructures[28]. The research partners in this project have been amongst the first in Australia to successfully use and analyse electronic patient data from general practice and evaluate patient outcomes in both cross sectional and longitudinal studies[21, 23]. 


\section{Sample size considerations}

Current estimates suggest that the study will be centred on data from over 350 general practices. This will provide sufficient scope to detect significant variation in practices across patient and general practice demographic domains.

\section{Analyses}

Data examination and analysis will be performed using Stata/MP 16 (StataCorp),[29] R v4.0.2 (R Core Team)[30], SAS 9.4 (SAS Institute) statistical software[31] and ArcGIS (Environmental Systems Research Institute)[32] for geo-spatial reporting. Statistical methods will include descriptive and inferential statistics (e.g., exploratory analyses using descriptive statistics, t-test for group comparisions) depending on the components of the project. Where spatial or temporal evaluations are required, stochastic (e.g., mixed models) and deterministic models (e.g., Bayesian structural time series) will be developed as per the study aims, and if applicable, incorporated into machine learning for building prediction models. The methods outlined in this research protocol will be structured according to the reporting of studies conducted using observational routinely collected health data (RECORD)[33]. The RECORD checklist deals specifically with real world research and evaluation using routinely collected data from electronic health records such as that provided by electronic general practice sources[33]. It represents the current best practice standard to ensure the robust reporting on non-interventional research using routinely collected health data[33].

The study will involve four components:

1) The building of a meaningful near real-time COVID-19 geo-spatial reporting framework and dashboard for decisionmakers at community, state and nation-wide levels, to identify and monitor emerging trends and the impact of interventions/policy decisions. This will include:

- Monitoring of the rates of COVID-19 testing in general practice.

- Examination of related general practice activities in the wake of COVID-19, particularly in relation to diagnostic (pathology and medical imaging) requests and medications.

- Key issues related to the identification of COVID-19 cases.

- Monitoring the impact of key risk factors (e.g., age, co-morbidities, smoking etc.)

- Identification of the impact of telehealth usage on the flexibility and responsiveness of general practice.

2) The generation of timely and critical evidence about the impact of the COVID-19 pandemic across the following care level dimensions:

- Clinical: Patient-level impacts based on:

Diagnostics (Most common COVID-19 symptoms; alarm flags for COVID-19, e.g., white blood cells, lymphocytes, platelet counts; risk factors, e.g., smoking, respiratory failure etc.);

Medications (The dangers of prescribing antiviral drugs e.g., drug-drug, drug-disease interactions; risks associated with chloroquine/hydroxychloroquine, ACE Inhibitors, aggravation of symptoms etc.); and,

Patient care (Special considerations for treatment in pregnancy, care of infants, use of nebulisers, recommendations for paediatric patients etc.).

- Population: What populations are being impacted, not only from a direct COVID-19 perspective, but from a regular care perspective (e.g., impact on chronic disease, preventative care and mental health).

- Business: What impact has the COVID-19 pandemic had on general practice (e.g., number and types of services, tests, medications etc.) from a business and financial perspective? Types of interaction with patients, telehealth vs 
face-to-face, how has this worked, what are the immediate and future implications? How has this impacted patient care on a clinical level and population level?

3) Develop a predictive geo-spatial analytics dashboard for timely, evidence-based decision-making at community, state and nation-wide levels and include:

- Visual representations of patient status and clinical environments that change dynamically in near real-time.

- Risk prediction tools based on statistical modelling incorporating demographics, co-morbidities, patient symptoms and risk factors.

- Incorporation of displays and decision support features, tailored to the needs and preferences of key recipients including GPs, PHNs and government health agencies.

4) Establish an evidence-based suite of general practice outcome measures required to monitor the quality and effectiveness of care related to incidence, prevalence, recovery and mortality.

\section{Patient and public involvement in research}

Each of the project partners have strong track records of collaborative research which includes engagement with patients and the public. The Macquarie University research team has an established Consumer Reference Group made up of consumer representatives who have contributed to the design, development and promulgation of research aims[34, 35]. The Consumer Reference Group was initiated following a national stakeholder forum (which brought together representatives from 14 stakeholder groups including patient organisations, clinicians and healthcare professionals) that outlined key patient safety challenges related to the utilisation of digital health and the diagnostic process[35]. The project will draw on established PHN stakeholder (patient and public) involvement using design thinking approaches to inform research questions, the design and conduct of studies and choice of outcome measures.

\section{Discussion}

\section{Research governance}

The Project will establish an operational and governance structure consisting of a:

a) Project Management Team (PMT) which will consist of representatives of the collaborating partners (Macquarie University, Outcome Health, Gippsland, Eastern Melbourne, and South Eastern Melbourne PHNs). The PMT will be responsible for the day-to-day functioning of the Project. It will draw on a team of clinical, technical and digital health analytics experts drawn from each of the collaborating partners, who will provide regular timely advice and guidance to generate meaningful robust, validated outcomes. This will include liaison with all stakeholder groups (general practice, nurses, managers and consumers).

b) Project Control Group that will consist of key representatives from the collaborating partners and the DHCRC with responsibility to oversee the Project's progress, priorities and direction. The Project Control Group will meet quarterly and be presented with interim reports for consideration during the course of the Project.

\section{Ethics and dissemination}

Outcome Health has received ethical approval from the Royal Australian College of General Practitioners National Research and Evaluation Ethics Committee (NREEC) 17 - 008 POpulation Level Analysis and Reporting (POLAR) GP 
data warehouse. The Macquarie University Human Research Ethics Committee (HREC) Medical Sciences Committee has granted approval to the project (Reference no. 5202067517176).

The operational objectives of the Project have been designed to contribute to the enhancement of quality general practice care in response to COVID-19. This will be achieved through the delivery of:

- The geo-spatial reporting framework will be co-developed and enhanced through feedback and design thinking mechanisms to address the impact of COVID-19 and the respective needs of all stakeholders. This will provide a foundation for enhanced care monitoring and planning across community, state and national level.

- The generation of robust evidence based on general practice data has enormous benefits for industry, health care providers and governments for timely decision-making and sound policy development.

- A predictive geo-spatial analytics dashboard will provide a dynamic tool to inform decisions about responses to COVID-19 along with other key areas of health (clinical and population).

- An evidence-based suite of general practice outcome measures to monitor the incidence, prevalence, recovery and mortality of responses to COVID-19.

Findings will be regularly communicated to GPs, PHNs, the community and nationally through near real-time snapshot reports. Findings will also be disseminated in peer-reviewed academic journals and presentations (national and international conferences and industry forums).

\section{Abbreviations}

\section{PHNs}

Primary Health Networks

GP

General practitioner

\section{PIMS-TS}

Paediatric Inflammatory Multisystem Syndrome

MIS-C

Multisystem Inflammatory Syndrome in Children

\section{DHCRC}

Digital Health Cooperative Research Centre

\section{HbA1c}

Glycated haemoglobin

PSA

Prostate specific antigen

\section{POLAR}

POpulation Level Analysis and Reporting

\section{RECORD}

Reporting of studies Conducted using Observational Routinely-collected Data

\section{PMT}

Project Management Team

\section{NREEC}

National Research and Evaluation Ethics Committee

HREC

Human Research Ethics Committee 


\section{Declarations}

\section{Declarations}

\section{Ethics approval and consent to participate}

Outcome Health has received ethical approval from the Royal Australian College of General Practitioners National Research and Evaluation Ethics Committee (NREEC) 17-008 POpulation Level Analysis and Reporting (POLAR) GP data warehouse. The Macquarie University Human Research Ethics Committee (HREC) Medical Sciences Committee has granted approval to the project (Reference no. 5202067517176).

\section{Consent for publication}

Not applicable.

\section{Avaliability of data and materials}

Not applicable.

\section{Competing interests}

The authors declare that they have no competing interests.

\section{Funding}

The project is funded by a Digital Health Cooperative Research Centre Partnership Project grant (DHCRC-0118). The funder had no role in the design of the protocol and the writing of the manuscript.

\section{Authors' contributions}

$A G, J L, C P, A M c, N W, R-A H, G F, C l, G S, J T, Z D, M K S, A P, S W, B W, T B, D M$ conceptualised and designed the work. AG, CP, $A M c, N W, R-A H, G F, C l, J T, Z D, M K S, A P, S W, B W$ were involved in the acquisition of data. AG, JL, CP, AMc, NW, R-AH, GF, $\mathrm{Cl}, \mathrm{GS}, \mathrm{JT}, \mathrm{ZD}, \mathrm{MKS}$, AP, SW, BW, TB, DM were involved in the analysis and/or interpretation of data. AG, JL, CP, AMc, NW, $\mathrm{R}-\mathrm{AH}, \mathrm{Cl}$ drafted the work. All authors critically revised the manuscript for important intellectual content and all authors approved the final manuscript.

\section{Acknowledgements}

Not applicable.

\section{References}

1. Altman M, Huang TT, Breland JY. Peer reviewed: Design thinking in health care. Prev Chronic Dis 2018;15.

2. World Health Organization. Diagnostic stewardship - A guide to implementation in antimicrobial resistance surveillance sites. Geneva: World Health Organizaion; 2016.

3. Reed S. Nuffied Trust Briefing. July 2020 - Resuming health services during the Covid-19 pandemic. What can the NHS learn from other countries? 2020 [Accessed 3 August 2020]. Available:

https://www.nuffieldtrust.org.uk/research/resuming-health-services-during-the-covid-19-pandemic-what-can-thenhs-learn-from-other-countries. 
4. Carr S. Missed and Delayed Diagnoses of Non-COVID Conditions - Collateral Harm from a Pandemic. ImproveDx Newsletter. 2020;7:1-7.

5. Mareiniss DP. The impending storm: COVID-19, pandemics and our overwhelmed emergency departments. Am J Emerg Med. 2020;38:1293.

6. Zhang Y, Liu J, Scott A. Who is avoiding necessary healthcare during the COVID-19 pandemic? Research Insights.: Melbourne Institute: Applied Economic \& Social Research, The University of Melbourne; 2020 [Accessed 23 July 2020]. Available from:

https://melbourneinstitute.unimelb.edu.au/_data/assets/pdf_file/0019/3401821/ri2020n13.pdf.

7. Australian Bureau of Statistics. Household Impacts of COVID-19 Survey, 29 Apr - 4 May 2020. 2020 [Accessed 5 August 2020]. Available:

https://www.abs.gov.au/AUSSTATS/abs@.nsf/allprimarymainfeatures/50884FE62C2C85C0CA258576008061F0? opendocument.

8. Zhang Y, Liu J, Scott A. Who is avoiding necessary healthcare during the COVID-19 pandemic? Research Insights. Available at: https://melbourneinstitute.unimelb.edu.au/_data/assets/pdf_file/0019/3401821/ri2020n13.pdf Accessed: 23 July 2020: Melbourne Institute: Applied Economic \& Social Research, The University of Melbourne; 2020.

9. Know Pathology. Why Australians are being urged not to delay pathology tests during COVID-19 restrictions 2020 [Accessed 12 May 2020]. Available: https://knowpathology.com.au/2020/04/23/do-not-delay-pathology-testsduring-covid-19-restrictions/? utm_source=ActiveCampaign\&utm_medium=email\&utm_content=Spread+the+word++Don+t+Skip+Tests\&utm_campaign=KPKH+April+2020+newsletter.

10. Victoria State Government Health and Human Services. Coronavirus COVID-19 daily update 2020 [Accessed 20 August 2020]. Available: https://www.dhhs.vic.gov.au/coronavirus-covid-19-daily-update.

11. Lam RPK, Hung KKC, Lau EHY, et al. Clinical, Laboratory, and Radiological Features Indicative of Novel Coronavirus Disease (COVID-19) in Emergency Departments-A Multicentre Case-Control Study in Hong Kong. J Am Coll Emerg Physicians Open. 2020. DOI:10.20944/preprints202005.0285.v1.

12. Mahase E. Covid-19: What do we know about "long covid"? Br Med J 2020;370.

13. Greenhalgh T, Knight M, Buxton M, et al. Management of post-acute covid-19 in primary care. Br Med J 2020;370.

14. Feldstein LR, Rose EB, Horwitz SM, et al. Multisystem inflammatory syndrome in US children and adolescents. N Engl J Med. 2020;383:334-46.

15. Kidd M. Australia's primary care COVID-19 response. Aust J Gen Pract 2020;49.

16. Desborough J, Hall SL, de Toca L, et al. Australia's national COVID-19 primary care response. Med J Aust. 2020;213:104-6.

17. Knapp J. The importance of face-to-face consultations during the coronavirus pandemic: Why and how for general practice. 2020 [Accessed May 20 2020]. Available: https://nwmphn.org.au/news/importance-face-faceconsultations-coronavirus-pandemic-general-practice/.

18. Australian Government Department of Health. Coronavirus (COVID-19) Primary Care Package - Practice Incentive Payments. 2020 [Accessed 17 Aug 2020]. Available:

https://www.health.gov.au/sites/default/files/documents/2020/03/coronavirus-covid-19-primary-care-packagepractice-incentive-payments.pdf.

19. Pearce C, McLeod A, Rinehart N, et al. What a Comprehensive, Integrated Data Strategy Looks Like: The Population Level Analysis and Reporting (POLAR) Program. In: Ohno-Machado L, Seroussi B, editors. MedInfo 2019: Health and Wellbeing e-Networks for All. Amsterdam: IOS Press; 2019. pp. 303-7. 
20. Roberts JP, Fisher TR, Trowbridge MJ, et al. A design thinking framework for healthcare management and innovation. Healthcare. 2016;4:11-4.

21. Sezgin G, Georgiou A, Hardie RA, et al. Compliance with pathology testing guidelines in Australian general practice: protocol for a secondary analysis of electronic health record data. BMJ Open. 2018;8:e024223.

22. Imai C, Hardie R-A, Franco GS, et al. Harnessing the potential of electronic general practice pathology data in Australia: an examination of the quality use of pathology for type 2 diabetes patients (In press). Int J Med Inform 2020.

23. Hardie R-A, Sezgin G, Imai C, et al. Enhancing patient outcomes through evaluation of the appropriateness and quality use of pathology in general practice: a report to the Department of Health Quality Use of Pathology Program. Sydney: Australian Institute of Health Innovation; 2020.

24. Central and Eastern Sydney Primary Health Network. [Accessed 18 September 2020]. Available: .

25. South Western Sydney Primary Health Network. Our Region [Accessed 18 September 2020]. Available: .

26. Pearce C, Shearer M, Gardner K, et al. A division's worth of data. Aust Fam Physician. 2011;40:167.

27. Pearce C, McLeod A, Patrick J, et al. Coding and classifying GP data: the POLAR project. BMJ Health \& Care Informatics 2019;26.

28. Bhatia RS, Levinson W, Shortt S, et al. Measuring the effect of Choosing Wisely: an integrated framework to assess campaign impact on low-value care. BMJ Qual Saf. 2015;24:523-31.

29. Stata/MP. [Accessed 11 September 2020]. Available: https://www.stata.com/statamp/.

30. The R Project for Statistical Computing. [Accessed 11 September 2020]. Available: www.r-project.org.

31. SAS Institute. [Accessed 11 September 2020]. Available: https://www.sas.com/en_au/home.html.

32. ArcGIS Enterprise. Copyright Information [Accessed 11 September 2020]. Available: https://enterprise.arcgis.com/en/portal/10.3/use/copyright-information.htm.

33. Langan SM, Schmidt SA, Wing K, et al. The reporting of studies conducted using observational routinely collected health data statement for pharmacoepidemiology (RECORD-PE). Br Med J. 2018;363:k3532.

34. Dahm M, Brown A, Martin D, et al Dahm M, Brown A, Martin D, et al. Interaction and innovation - practical strategies for inclusive consumer-driven research in health services BMJ Open 2019;9.

35. Dahm M, Georgiou A, Herkes R, et al. Patient groups, clinicians and healthcare professionals agree - all test results need to be seen, understood and followed up. Diagnosis. 2018;5:215-22.

\section{Supplementary Files}

This is a list of supplementary files associated with this preprint. Click to download.

- RECORDChecklist.docx 